\title{
Crowd behaviour modelling developments through mixed integer programming: the case of airport queue management
}

\author{
Mahdi Doostmohammadi \\ Dept. of Management Science \\ University of strathclyde \\ Glasgow, UK \\ Email: m.doostmohammadi@strath.ac.uk
}

\author{
Emmanuel Fragniere and Rosanna Holdsworth \\ School of Management \\ UAS Western Switzerland and University of Bath \\ Sierre, Switzerland \\ Email: emmanuel.fragniere@ hevs.ch
}

\begin{abstract}
Crowd behaviour is difficult to predict and might not be easy to translate. A number of mathematical and psychological models are proposed in the literature to investigate crowd behaviour. In this paper, we exploit mixed integer programming to model crowd behaviour with multiple time periods. This research improves upon methods by Breer et al. (2015) for determining the number of active agents and solving the problem of reducing this number by controlling reputations in a single period, under the added assumption of a reputation model of interactions (Granovetter, 1978). Thus, this paper goes on to extend the single period reputation control problem and solution to the case of multiple time periods. This class of problems requires a mixed integer program to be solved several times with a varying constraint and a varying number of variables. This model is then supported by a promising case study of queue management at airport security gates.
\end{abstract}

Index Terms-crowd behavior; decision aid tool; mixed integer programming; reputation control model; threshold model;

\section{INTRODUCTION}

Passenger queues at airports represent an important challenge today due to unexpected overall behaviour like panic or the outbreak of violence. Many of the models arising from the scientific literature address these issues. A large family of these models is based on simulation techniques such as the wellknown agent-based approach. This latter approach can be considered as a trial and error one. Given a specific layout and predetermined human flows, adding a disturbance to the model enables to see how the situation might deteriorate. These models are usually descriptive. They tend to explain past cases like the love parade in Germany that ended up with deaths and many injured participants. Incidentally, this topic of crowd behaviour has become an important research topic for the EU.

In this paper, we want to go a step further as compared to these more classical models that are purely descriptive. Even if descriptive models are good to learn about behavioural patterns, a decision aid model can help to provide an appropriate response to contain a contagion (herd effect) that could lead to dramatic outcomes. In this research, we thus try another approach that is more decision making orientated. We rely on the threshold model as first introduced by Granovetter. It states that each agent be given a threshold corresponding to the number of other agents who would need to be taking part in an activity before they would join in. This model, when properly defined, can be translated into an MIP model and solved using branch and bound techniques. However, the original threshold model is static. In this paper we have developed, for the first time to our knowledge, a multi-stage formulation in order to capture the dynamic nature of crowd behaviour. The main contribution of this 
research is theoretical. It has consisted in formally defining human interactional constructs and translating them as well into mathematical expressions to properly capture real crowd phenomena. The paper is organized as follows. In Section 2, we present a brief literature review about the different kinds of approaches that addresses crowd behaviour issues, we also describe the original threshold model as proposed by Granovetter. In Section 3, we present our methodological contribution, we explain how the threshold model has been adapted to airport queue behaviour and how it has been transformed to integrate multiple periods. In Section 4, an illustrative case study is developed to explain the type of results provided by our new model and how they differentiate from those produced by other approaches (in-deed they all offered different perspectives and cannot thus be strictly compared for same crowd configurations and data sets).

\section{LITERATURE REVIEW}

Crowd behaviour has been extensively studied by researchers during the last decade. Social influence in a general manner has been the subject of study in many different fields. For instance the phenomena of herding, defined as "the alignment of the thoughts or behaviours of individuals in a group (herd) through local interaction and without centralized coordination" by [5]. This behaviour can cause stock market bubbles, influence political opinions and cause the formation of mass riots. So understanding it has been of interest not only to psychologists, but also economists and sociologists, amongst others [5]. Social influence doesn't just apply to emotions, it can also affect decision-making. Mathematically this can be modelled as agents moving between discrete states depending on the states of other agents. Typically these models consider binary states, for instance agents choosing between being "active" or "passive". There are many models to choose from in this area, originating from many different academic disciplines. For instance, the threshold model, the independent cascade model, the SIR model, hazard rate models and models of pluralistic ignorance. However, as [6] points out, although these models are all trying to describe the same behaviour they are often irreconcilable due to the assumptions made by each. The independent cascade model requires a graph of connections between the agents and a probability of transmission $p$. At the initial time step certain agents are set to "infected", these are the "seeds", and at each subsequent time step newly infected agents infect each adjacent uninfected agent with probability $p$. The weighted cascade model is similar, but sets the probability of agent $i$ infecting agent $j$ to be $\frac{1}{d}$, where $d$ is the in-degree of agent $j$ i.e. the number of agents who could infect $j$. These types of model can be used, for instance, to predict the spread of infections, the popularity of a new trend and how quickly a rumour spreads. The threshold model was introduced by Granovetter in [4], proposing that each agent be given a threshold corresponding the number of other agents who would need to be taking part in an activity before they would join in. Granovetter used the following example to show how this model could be used to explain the unstable nature of the size of a riot. Suppose there are one hundred agents each with a threshold between 0 and 99 (one agent with 0 threshold, one with 1, one with 2 and so on). The 0 -threshold agent will start to riot as they do not require any other agents to be rioting in order to do so themselves. Therefore there will be one rioting agent, and so the 1-threshold agent will also start to riot, and so on until all agents have joined the riot. However, suppose the distribution of thresholds is instead: one agent of threshold 0 , two of threshold 2 , one of threshold 3 and so on (so the change from the previous example is just one agent increasing their threshold by 1 ). Then the 0 -threshold will again start to riot, but no other agents will join them as all other thresholds are above 1. The book [2] shows how threshold models of collective statemaking has expanded since Granovetter's original paper. The general threshold model explained here allows for interactions between agents to differ certain agents will have more of an influence on a particular agent's state than others. The problem of how to control the number of active agents has been studied for many of the different models of binary states. For instance, [3] considered the question 
of maximizing influence in a network under both cascade models by choosing the seeds, which is an NP-hard problem. This paper proposed a genetic algorithm to solve this problem and compared it to the performance of a number of known heuristics, using data from social networking sites. [2] addresses the problem of mob control under the threshold model - how to minimize (or maximize) the number of active agents given different constraints and assumptions. This book [2] also states and solves a multiperiod control problem of introducing "provokers" to maximize active agents.

\section{Multiple-Period Reputation CONTROL PROBLEMS}

Suppose a group of agents who each have a known reputation and known threshold and are influenced by every other agent in the group according to their reputations. Assume there is at least one ringleader in this group (an agent with a threshold of 0 ), so that at the collective behaviour equilibrium the group will have at least one active member. Suppose an outside force acting on individual agents in the group can lower the reputations of individuals for a certain cost. The reputation control problem is to choose which reputations should be changed, and by how much. To mathematically model this problem, let $N=\{1,2, \ldots, n\}$ is the set of agents in the system, $\theta \in[0,1]$ is the threshold of agent $i$, $m_{i}$ is the minimum reputation of agent $i, r_{i}^{0}$ is the reputation of agent $i$ in the initial state, $r_{i}^{1}$ is the reputation of agent $i$ after all changes have been made to reputations and reputations have been normalised (the final state), and $\hat{r}_{i}^{0}$ is the cumulative reputation of agent $i$ in the initial state. Furthermore, assume $k^{*}$ is the maximum number of active agents allowed and $R_{k^{*}}$ is the sum of reputation changes that need to be made to the first $k^{*}$ agents in order for agent $k^{*}+1$ 's cumulative reputation to equal its threshold, define by the following equation:

$$
R_{k^{*}}=\frac{\hat{r}_{k^{*}+1}^{0}-\theta_{k^{*}+1}}{1-\theta_{k^{*}+1}}
$$

Define binary decision variables $x_{i}$ as $x_{i}= \begin{cases}1, & \text { if a change is made to agent } i \text { 's reputation } \\ 0, & \text { otherwise }\end{cases}$ Then the single-period reputation control problem is modelled as

$$
\begin{aligned}
\operatorname{Min} & \sum_{i=1}^{k^{*}} c_{i} x_{i} \\
\text { s.t. } & \sum_{i=1}^{k^{*}}\left(r_{i}^{0}-q^{i}\right) x_{i}>R_{k^{*}} \\
& x_{i} \in\{0,1\}
\end{aligned}
$$

where $c_{i}$ and $q_{i}$ are the cost of changing agent $i$ 's reputation to $m_{i}$ and $i$ 's minimum reputation respectively.

To extend the optimization problem of the singleperiod reputation control model (1) to the multipleperiod case we can take inspiration from the way the Warehouse Location Problem can be extended to the multiple-period case. An indicator variable is introduced for whether an agents' reputation is changed for the first time in a certain period. When dealing with more than one period we must include all agents in the optimization (whether they are one of the first $k_{t}$ in period $t$ or not). This means the new variables $u_{i t}$ and $w_{i t}^{k}$ (defined above) are needed to ensure the correct agents are optimised over.

Let $a_{i t}$ be the cost to change agent $i$ 's reputation in period $t, f_{i t}$ be the startup cost to be paid when agents $i$ 's reputation is changed in period $t$ but not in period $t-1$, and $R_{k t}=\frac{\hat{r}_{k_{t}+1}^{\mathrm{C}}-\theta_{k+1, t}}{1-\theta_{k+1, t}}$. Define the variables of the optimization problem as

$$
\begin{aligned}
& x_{i t}= \begin{cases}1, & \text { if agent } i \text { 's reputation is changed in period } t \\
0, & \text { otherwise }\end{cases} \\
& y_{i t}= \begin{cases}1, & \text { if agent } i \text { 's reputation was unchanged in period } t-1 \\
0, & \text { if agent } i \text { 's reputation was changed in period } t-1\end{cases}
\end{aligned}
$$

The multi-period reputation control problem 
is:

$$
\begin{aligned}
\operatorname{Min} & \sum_{i=1}^{T} \sum_{i=1}^{n} a_{i t} \cdot x_{i t}+\sum_{i=1}^{T} \sum_{i=1}^{n} f_{i t} \cdot y_{i t} \\
\text { s.t. } & \sum_{i=1}^{n} w_{i t}^{k_{t}} \cdot\left(r_{i t}^{0}-m_{i t}\right) \cdot x_{i t}>R_{k t}, \forall t \in P \\
& x_{i t} \leq u_{i t}, \forall i \in N, t \in P \\
& y_{i t} \leq x_{i t}, \forall i \in N, t \in P \\
& x_{i t}-x_{i, t-1} \leq y_{i t}, \forall i \in N, t \in P \\
& x_{i t}, y_{i t} \in\{0,1\}, \forall i \in N, t \in P
\end{aligned}
$$

The first constraint ensures that the reputation reductions meet the requirement for moving agent $k_{t}+1$ below the diagonal for every $t$ in $P$, where $w_{i t}^{k_{t}}$ ensures that only reductions to the first $k_{t}$ agents in each period count towards this sum (as these are the only reputations that influence the cumulative reputation of agent $k_{t}+1$. The second constraint ensures that only reputations of agents who are in the system in period $t$ can be changed in period $t$. The third constraint implies that if an agents' reputation is being changed for the first time in period $t$, then this agents reputation is being changed in period $t$ (the startup cost only needs to be paid if that agent's reputation is being changed in that period). The fourth constraint implies that an agents' reputation is being changed for the first time in period $t$ only if it is being changed in period $t$ and if it was not changed in period $t-1$.

Solving the above optimization for $k_{t}=k_{t}^{*}, \forall t \in$ $P$ provides a starting point for the solution of the Reputation Control Problem, and an upper bound on the value of the objective function if the problem is feasible, just as in the single-period case. Following the same logic as in the single-period case, the only values of $k_{t}$ that need to be tried are those that satisfy $R_{k_{t} t} \leq R_{k_{t}^{*} t}$, and for which agent $k_{t}$ does not share a threshold with an earlier agent. Let these values of $k_{t}$ form the set $K_{t}$. Then the optimization above must be solved once for each combination of elements from the sets $K_{t}$.

In an ideal world we would want to model the formulation of this problem to include the constrain that if an agent becomes active in period $t$ then they will also be active in period $t+1$. However, the complicated nature of the relationship between $k_{t}$ and the number of active agents after reductions are made means there is no easy way to include this in the optimization problem formulation. Further work into how this could be modelled may be an interesting area of study.

\section{CAse Study}

Imagine there are twenty-two passengers waiting in line to get through passport control. There is a family of four, two couples, a group of six friends, and the remaining eight passengers are travelling alone. Suppose the passengers are lined up as shown in the illustration below. Groups of passengers who are travelling together are indicated. The queue is moving very slowly and many of the passengers have already spent a long time queueing up meaning they are frustrated and bored. In addition, the person at the front of the queue is taking a long time to be processed, and some of the passengers behind are becoming increasingly agitated at the prospect of missing their flight as a result of the delay. Some of the passengers are travelling with others and may place more weight on the opinions of those they know than of strangers, thus placing this problem in the more general case of the threshold model. However, the reputation case can be applied to this example by assuming that the threshold of each passenger in a group is the same as every other member of the group, which would mean that all members of the group would riot simultaneously if this threshold is breached. Or, alternatively, by assuming that if one member of a group starts to riot then all the other members of the group instantaneously do so too due to the disproportionate influence on them by people they know. Making one of these assumptions means each group can be considered as a single agent and the group can be assigned a single threshold and a single reputation. It must also be assumed that the physical formation of the queue has no bearing on the influences between agents. 


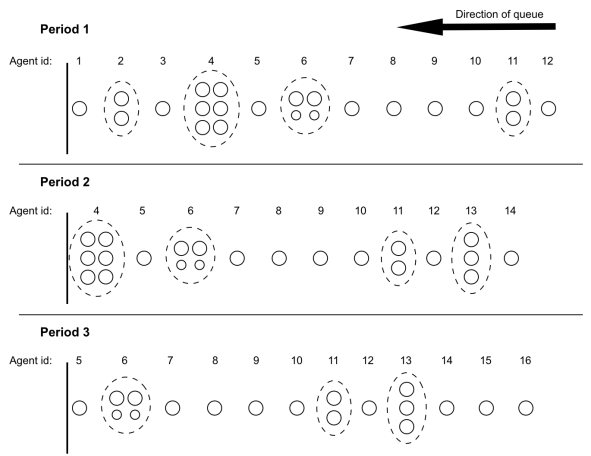

Illustration of the queue changing over three periods.

Assume the costs are the same as defined in Section III: $f_{i t}=2, \forall i \in N ; t \in P$ and $a_{i t}=$ $s_{i}$ (No. of adults), $\forall i \in N ; t \in P$ (we assume the size of the groups has not changed). Table 1 describes the changes to reputations, thresholds and the order id of agents over three periods.

\begin{tabular}{|c|c|c|c|c|c|c|c|c|c|c|}
\hline \multirow[b]{2}{*}{ Agent id } & & $\mathrm{Per}$ & $\begin{array}{l}\text { int thr } 1 \\
\text { iod }\end{array}$ & & \\
\hline & $s_{i}$ & Order id & $r_{i}^{1}$ & $\theta_{i}^{1}$ & Order id & $r_{i}^{2}$ & $\theta_{i}^{2}$ & Order id & $r_{i}^{3}$ & $\theta_{i}^{3}$ \\
\hline 1 & 1 & 11 & 0.05 & 0.8 & का & - & - & - & - & - \\
\hline 2 & 2 & 3 & 0.1 & 0.05 & - & - & - & - & - & - \\
\hline 3 & 1 & 4 & 0.05 & 0.1 & - & - & 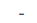 & - & - & - \\
\hline 4 & 6 & 5 & 0.3 & 0.2 & 8 & 0.3 & 0.5 & 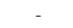 & - & - \\
\hline 5 & 1 & 1 & 0.05 & 0 & 1 & 0.05 & 0 & 1 & 0.0625 & 0 \\
\hline 6 & 2 & 12 & 0.1 & 1 & 11 & 0.1 & 1 & 12 & 0.125 & 1 \\
\hline 7 & 1 & 6 & 0.05 & 0.5 & 3 & 0.05 & 0.1 & 4 & 0.0625 & 0.05 \\
\hline 8 & 1 & 7 & 0.05 & 0.5 & 4 & 0.05 & 0.1 & 5 & 0.0625 & 0.05 \\
\hline 9 & 1 & 8 & 0.05 & 0.5 & 5 & 0.05 & 0.1 & 6 & 0.0625 & 0.1 \\
\hline 10 & 1 & 2 & 0.05 & 0 & 2 & 0.05 & 0 & 2 & 0.0625 & 0 \\
\hline 11 & 2 & 9 & 0.1 & 0.75 & 6 & 0.1 & 0.2 & 7 & 0.125 & 0.1 \\
\hline 12 & 1 & 10 & 0.05 & 0.75 & 7 & 0.05 & 0.4 & 8 & 0.0625 & 0.3 \\
\hline 13 & 3 & - & - & - & 9 & 0.15 & 0.7 & 9 & 0.1875 & 0.5 \\
\hline 14 & 1 & - & - & - & 10 & 0.05 & 0.7 & 9 & 0.0625 & 0.5 \\
\hline 15 & 1 & . & . & 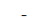 & - & - & & 3 & 0.0625 & 0 \\
\hline 16 & 1 & 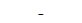 & 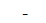 & 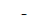 & 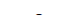 & 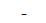 & - & 11 & 0.0625 & 0.8 \\
\hline
\end{tabular}

If we take each period of this case in isolation and apply the solution method developed for the single-period reputation control problem with fixed number of active agent to each (solved using R), then we find the solutions $x_{t}$ to be:

$$
x_{1}=(0,1,0,0,0,0,0,0,0,0,0,0,0,0,0,0)
$$

i.e. removing agent 2 . The objective function has a value of 4 .

$$
x_{2}=(0,0,0,0,1,0,0,0,0,0,0,0,0,0,0,0)
$$

i.e. removing agent 5 . The objective function has a value of 3 .

$$
x_{3}=(0,0,0,0,0,0,0,0,0,1,0,0,0,0,1,0)
$$

i.e. removing agents 10 and 15 . The objective function has a value of 6 . So if we took the solution of the multi-period reputation control problem with fixed number of active agents to be the combination of these solutions the cost would be 13 . The optimal answer to the multi-period reputation control problem for the airport queue example is: $x=(0,0,0,0,1,0,0,0,0,1,0,0,0,0,0,0)$ i.e. removing agents 5 and 10 in every period. The objective function has a value of 10 . Therefore this solution is an improvement to performing the single-period reputation control problem for each period.

\section{CONCLUSiON}

Consequently, the added value of such an approach is that it can be used in an ex-ante or anticipative mode. Indeed the advantage of the detailed mathematical formulation is that specific and complex queue configurations can be studied and modelled in advance with a precise predefined granularity. Risk responses and dispositions can then be elaborated and trained before running the queue process for real. For instance, in the developed case study, based on the different model instances optimized, we are able to precisely advise on the relevant number of trained staff that must be added to deal with a given queue system configuration taking into account possible crowd overflows. We indeed posit that crowd risks are driven by emotions and solely trained people (not technology) can contain them using their skills. We hope that this contribution will bring a different, although relevant, approach to crowd risk management that will complement the other existing approaches.

\section{REFERENCES}

[1] BREER, V. V., NOVIKOV, D. A. \& ROGATKIN, A. D. (2015) Models of Collective Threshold Behaviour in Control Problems of Ecological-Economic Systems. In GameTheoretic Models in Mathematical Ecology. New York: Nova Science Publishers. 
[2] BREER, V. V., NOVIKOV, D. A. \& ROGATKIN, A. D. (2017) Mob Control: Models of Threshold Collective Behaviour, Cham: Springer.

[3] BUCUR D. \& IACCA G. (2016) Influence Maximization in Social Networks with Genetic Algorithms. IN: SQUILLERO G., BURELLI P. (Eds) Applications of Evolutionary Computation, EvoApplications 2016 (Lecture Notes in Computer Science Vol. 9597), pp379-392, Cham: Springer.

[4] GRANOVETTER, M. (1978) Threshold Models of Collective Behaviour. The American Journal of Sociology. Vol. 83 (No. 6), pp1420-1443.

[5] RAAFAT, R. M., CHATER, N. \& FRITH, C. (2009) Herding in humans. Trends in Cognitive Sciences. Vol. 13 (No. 10), pp420-428.

[6] WATTS, D. J. (2017) Should social science be more solution-oriented? Nature Human Behaviour. Vol. 1 (No. 1). 